\title{
Design of a Mobile Campus Portal in Big Data Environment
}

\author{
Dandan Hong, Zhaozhong Fan, Junfeng Luo, Mo Xu, Zhihai Suo \\ Data and Information Centre of Xi'an Jiaotong University, Xi'an, China \\ Email:ddhong@mail.xjtu.edu.cn,zzfan@mail.xjtu.edu.cn,luojf@mail.xjtu.edu.cn, \\ mxu@mail.xjtu.edu.cn,zhsuo@mail.xjtu.edu.cn
}

Received 12 April 2016; accepted 24 May 2016; published 30 May 2016

\begin{abstract}
In order to fulfill the requirements of mobile computing and big data environments, we design a mobile campus portal (MCP) providing mobile information services for users in both browser/ server and client/server modes. We present the topological structure of MCP, system infrastructure, application architecture and the data management (such as data integration, data format translation, data security control and so on) of our mobile campus portal in this paper. After online running in the past two years, our MCP has received good feedbacks from mobile users. In the future, we will focus on the research of data-mining technologies in our big data environment.
\end{abstract}

\section{Keywords}

Big Data, Mobile Computing, Campus Portal, Data Integration, JSON

\section{Introduction}

With the development of smart mobile devices and big data technologies, mobile applications have become efficient tools for information sharing. Mobile campus applications in the educational industry have attracted more and more researchers.

For example, [1] has developed a mobile learning system based on mobile phone. This mobile learning system provides an interactive teaching platform between teachers and students. Learners can study online classes at any time, any place with the help of mobile devices. [2] proposed a mobile learning application towards Android smart phone and blackberry. This mobile application provides both client-side e-learning service for students and service-side interface sharing service.

For campus portal, there are tremendous applications be-sides e-learning, e.g., administrative management, re-search projects management, teachers and staffs management, students management, etc. Therefore a comprehensive mobile campus portal is needed in order to provide more mobile services for campus users.

In this paper, a mobile campus portal (MPC) is developed based on six-layer architecture. It combines the B/S (Brower/Server) and C/S (Client/Server) modes together. The topological structure is shown in Figure 1. It ensembles and provides extensive campus mobile applications for campus services or managements to meet the

How to cite this paper: Hong, D.D., Fan, Z.Z., Luo, J.F., Xu, M. and Suo, Z.H. (2016) Design of a Mobile Campus Portal in Big Data Environment. Int. J. Communications, Network and System Sciences, 9, 145-151.

http://dx.doi.org/10.4236/ijens.2016.95013 


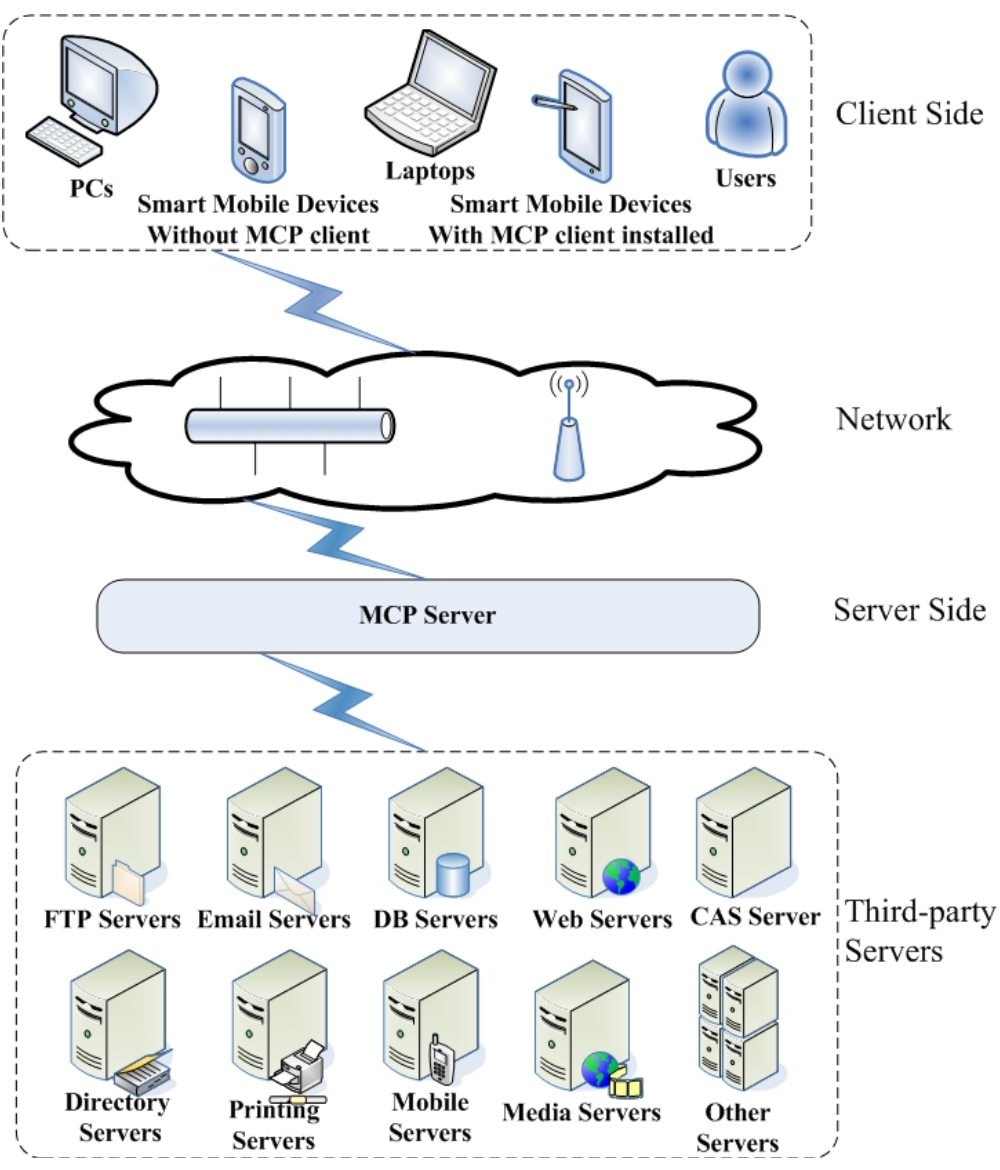

Figure 1. The topological graph of MCP.

requirements of educational big data environment in our university.

\section{Introduction to Big Data}

Big data research has been a hot topic nowadays, more and more researchers start to pay attention to this concept. For example, [3] defines twelve dimensions of big data concept towards higher education institutions. [4] elaborates a flexible system architecture based on the service-oriented specification to support social interactions in campus environments. This phenomenon shows that big data concept and technologies have already been integrated into the educational industry. Federal Big Data Working Group is aiding the implantation of Big Data applications in government and science communities [5], including educational industry. [6] defined big data in 6 Vs (Variety, Volume, Velocity, Variability, Complexity and Value). Khan defined big data in 7Vs (Volume, Velocity, Variety, Veracity, Validity, Volatility and Value) [7]. Al-though these definitions of big data are different, all re-searchers have in common with the $5 \mathrm{Vs}$ of big data: volume, variety, velocity, veracity and value [8]-[10]. In this paper, we will design an effective mobile campus portal by implementing the 5Vs of big data concept.

The rest of the paper is organized as follows. Section 2 presents the system infrastructure overview and topological structure of MCP from design aspect. Section 3 presents the application architecture of the MCP and the functions of each module. Section 4 discusses on how we implement the five dimensions of big data concept in MCP (Volume, Velocity, Variety, Veracity and Value). Section 5 concludes this paper with our future work.

\section{Infrastructure of MCP}

Based on the characteristic of mobile smart devices and educational big data concept, we design the infrastructures of MCP as follows. 


\subsection{Topological Structure}

On the client side (as shown in Figure 1), users can access the information provided by MCP in various ways.

- Firstly, if the client application (iOS or Android) is installed successfully in the smart mobile device, mobile user can access mobile service provided by MCP and the user-interactive experience provided by smart mobile device itself. Meanwhile, the user's context information (such as proximity, communication history and so on [5]) can also be sent back to the MCP server for the usage of data mining in educational big data.

- Secondly, if the MCP client application is not in-stalled in the smart mobile device, user can access the mobile service provided by MCP with the help of mobile browser installed in the smart mobile de-vice under B/S mode. That is why we adopt the hybrid mode to develop our mobile campus portal. But the user-interactive experience may not be as good as the C/S mode.

- Finally, for the users who have no mobile devices with them, they can still access the information ser-vice provided by MCP under the help of web browser installed in PCs or laptops in B/S mode.

The detailed application requirements and modules provided by MCP will be explained in Section 4.3.

On the server side, MCP collects data from all the third-party systems and provides data services for campus users. As shown in Figure 1, there are various kinds of third-party servers providing information sharing for MCP. Campus users do not need to log on each individual sys-tem to access information. They can request information services as much as possible by a single log in the MCP, which reduces the possible troubles caused by frequent log-in operations. The details on data processing in MCP will be explained in Section 4 .

For security, MCP uses the Central Authentication System (CAS) to keep the platform safe and transform data by SSL mode. All these two methods can guarantee all the information that the users get is reliable and authentic, which will be explained in Section 4.

\subsection{System Layers}

Based on the topological structure of MCP shown in Figure 1, we build the layer infrastructure by six layers logically. Each layer interacts with the others and has its main function. The layer infrastructure is shown in Figure 2.

- Base layer includes the hardware and software that MCP relies on, such as networks, servers, firewalls, middleware, server OS and the other required soft-wares.

- Data integration layer completes data collection and data integration, including structured data in RDBM (relational database system) and unstructured data in the other systems.

- Data sharing layer includes the basic data, processed data and statistical data provided by server for the client's usage, in order to meet the mobile users' requirements in MCP. Beside this, all the shared data are formed in JSON (JavaScript Object Notation) format which is a very lightweight data structure that both Android and iOS platforms can work effectively with it.

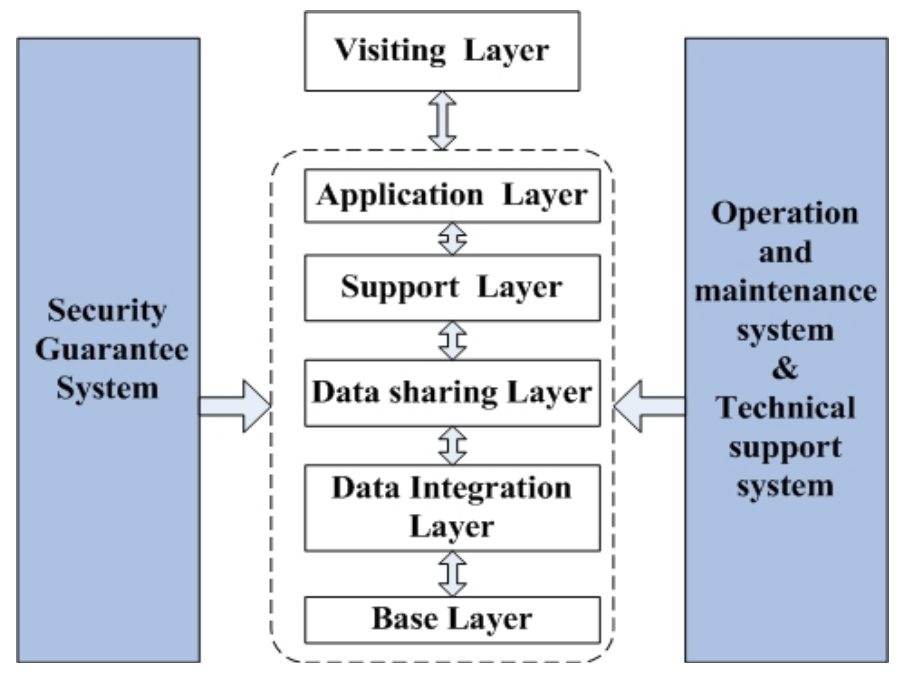

Figure 2. The layer infrastructure for MCP. 
- Support layer includes two kinds of supporting ser-vices. For the public services, the CAS, payment management will be provided for the upper layer. For the application services, the components involving log management, GIS service, message management, searching management and so on are provided for the upper layer.

- Application layer includes all the mobile applications provided for mobile users, such as financial application, online classes application, mobile teaching application, mobile GIS application, etc. Mobile users can access these mobile applications by their smart device with the MCP client installed.

- Visiting layer is the entry of all applications. It consists of mobile clients, which can interact with the underlying servers in order to provide information services for mobile users.

Combing the topological and layer infrastructures of MCP, we develop the application architecture in Section 3.

\section{Application Architecture of MCP}

At the application level, MCP provides five types of mo-bile application modules for users including teaching management, administrative management, financial management, research management and public services. These module structures are shown in the Figure 3.

\subsection{Teaching Management Module}

This module consists of six child-modules, they are specified in the followings.

- Students enrolment. This module provides services for publishing the enrolment information about undergraduate and graduate students, querying the en-try scores and enrolment information in the past years.

- Curriculum management. This module provides services of querying curriculum schedules based on the user's role. For example, student can query the information of their courses bases on his/her student ID. Teachers can query the schedules of the courses that they teach based on his/her employee ID.

- Staff homepages. This module publishes the basic information of all the staffs in the university, such as the contact information, research areas, enrolment in-formation, personal homepage addresses and so on.

- Online classes. This module provides the online learning interface for online public courses. All the courses are categorized by their subjects. Users can study these online classes for free.

- Mobile library. This module provides services for querying academic publications, library information and ordering books online.

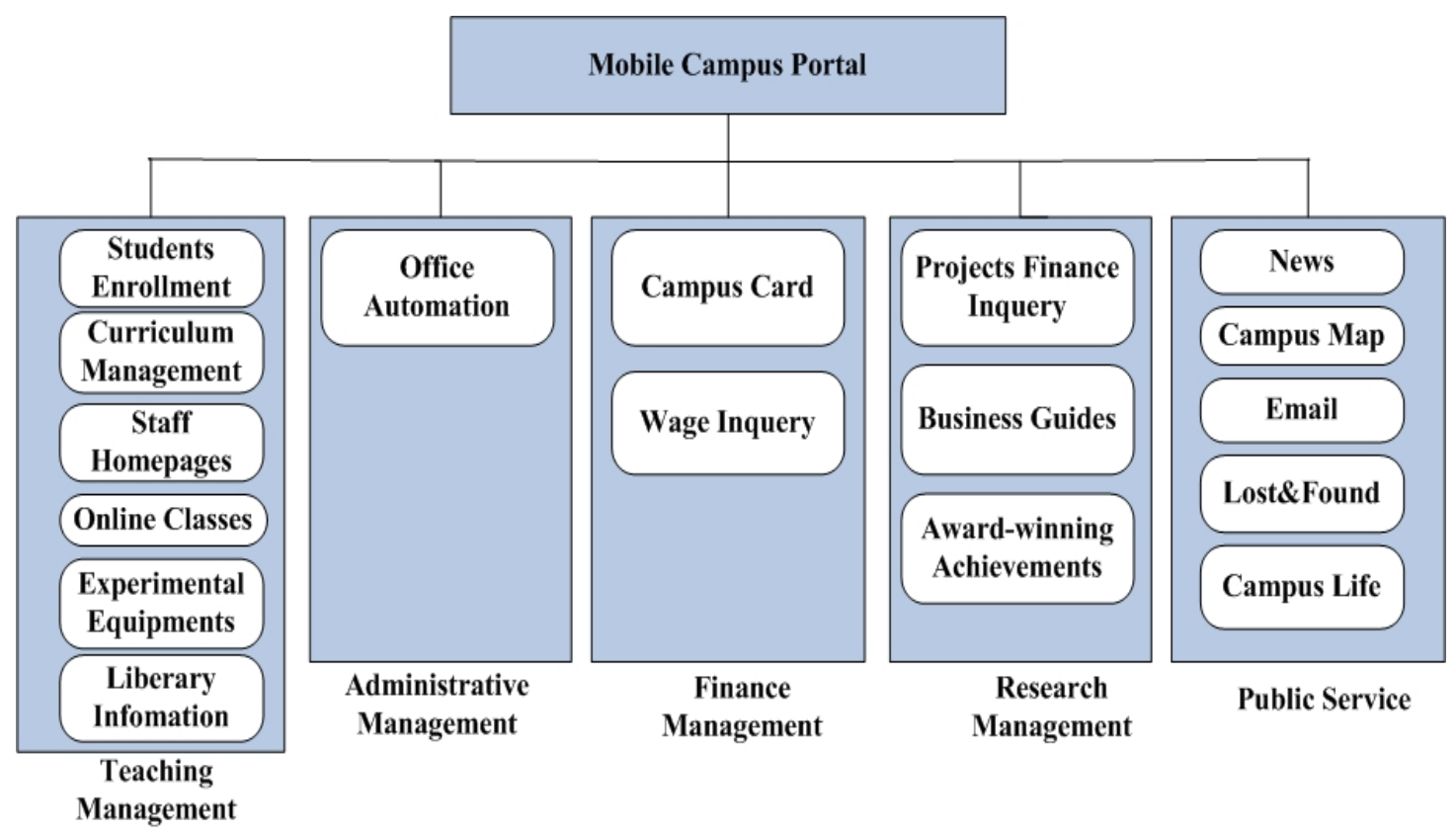

Figure 3. The application architecture of MCP. 
Experimental equipments. This module provides services for online ordering the equipment and querying the related information. Due to the real-name authentication for this function, the users are required to register before using this function.

\subsection{Administrative Management Module}

This module provides the mobile OA (Office Automation) for campus users to query different categories of announcements or notices relating to the administrative management. For the information with high level of confidentiality, users are required to register and assigned proper authorities according to their roles.

\subsection{Financial Management Module}

This module provides two main child-modules for campus users. Campus card module provides services for revising the basic information of campus ID card, and querying the balance and consumption records in the ID card. Wage inquiry module provides services of wage inquiry. Users who desire to use these functions must be authenticated successfully by the CAS (Central Authentication System).

\subsection{Research Management Module}

This module provides three services for researchers. After authenticated, the users can query the information on finance, awards and guides related to his/her research projects. CAS authentication is required for security reason.

\subsection{Public Service Module}

This module provides five child-modules. News module publishes all the campus news relating to the students, staffs, university developments, etc. Campus-map module provides services of campus map inquiry and the related functions, e.g. buildings localization. Email module provides services for email receiving and sending. The users are required to register on CAS system before using this service. Campus life module provides services for campus life such as dormitory inquiry, lost \& found, guides for freshman, electronic offers and so on.

\section{Implementation of Big Data in MCP}

To meet the requirements of big data environment in cam-pus, our mobile campus portal implements the five dimensions of big data concept (discussed in Section 1.1) during the whole lifecycles of data processing. Data integration implements the volume and variety dimensions. Data translation implements the velocity dimension. Data security control implements the veracity dimensions. Data mining implements the value dimension.

\subsection{Data Integration for MCP}

"Volume" of big data means the size of data, which are collected from all kinds of sources. "Variety" of big data means that data is formed in different formats, e.g., structured data generated by RDBMS (relational database management system) and unstructured data. Because of the large amount of data existing in the web sites, information systems, mobile devices, video/audio files and the other electronic resources in our campus, data integration is highly essential. Therefore MCP should collect information from all resources to implement the volume and variety of big data. Currently MCP integrates data from five types of electronic resources which are shown in Figure 3.

\subsection{Data Translation Workflow}

Velocity dimension of big data concept focuses on the speed of data generating and responding. In order to keep data transmission in high speed in the big data environment, MCP collects and shares information by web ser-vice interfaces which are formed in JSON format. JSON is one of the most popular data interchange format [11]. It has a high rate of flexibility in combination with a light-weight structure and has benefits of standardization [3]. Using JSON format, we design a data translator for MCP server to collect different kinds of data from various re-sources in data integration period and sharing period. The whole data translation workflow is shown 


\section{in Figure 4.}

For the systems (resources) which can only provide shared views of databases, MCP server sets remote connections to the shared views, gets structured data and converts them into standard JSON format by data translator, then provides the formatted JSON interfaces to the upper layers as shown in Figure 4 (workflow No.1).

For the third-party systems which can provide interfaces in JSON format directly, MCP calls these interfaces and provides data services for the upper layers without any transformation as shown in Figure 4 (workflow No. 2).

For the third-party web service interfaces which only pro-vide XML format, data translator converts the XML for-mat into standard JSON format for the objects in the upper layers as shown in Figure 4 (workflow No. 3).

For the web systems without any interface, we use web-parsing technique to capture the related information directly from the web pages, transform them into JSON format and then provide services to the objects in the up-per layer as shown in Figure 4 (workflow No. 4).

\subsection{Security Control Mechanism}

Veracity dimension of big data means truthfulness, correctness and accuracy of data. This dimension requires that all data in the MCP must not be modified by non-authenticated users. Only authenticated and authorized users can collect and share information in MCP. So the security control is required.

- Content security control. Because of the CAS (Central Authentication System) of our university is already applied for many years, and it has the official user profiles of our university, MCP adopts it to authenticate campus users. Combined with the RBAC (Role-Based Access Control) method, users are as-signed authorities to access the resources according to their roles. For example, the teachers can access and check information on their monthly salaries or research funds, but the un-authorized users (students or visitors) cannot access these information. The authorized students can access the systems for online courses, vacant classrooms search, campus recruitments, etc. The visitors can only access the public in-formation, such as campus news, online courses and so on.

- Transmission security control. Due to the special characteristics of mobile intelligent devices, many malicious users create fake WIFI hotspots, obtain the users' personal and sensitive information e.g., bank accounts, or revise the students' academic records [12]. Therefore, we use SSL protocol to encrypt data. On the application layer, we use encryption key to perform encoding/decoding for data between servers and clients, in order to protect the mobile data [13]. As stated in [14], SSL can be considered as a key solution to the fishing attack. Meanwhile, the mobile applications with SSL can also be protected against from the Manin-the-Middle Attack (MIMA) [13].

\subsection{Data Analysis and Mining}

Data analysis and mining is the most difficult step of big data processing, which can implement the value dimension. "Value" here means extracting valuable information from all the data that our MCP collected and generated. Currently our MCP provides the e-learning analysis of students. The other analysis such as e-health analysis of students and staffs will be developed in our future work.

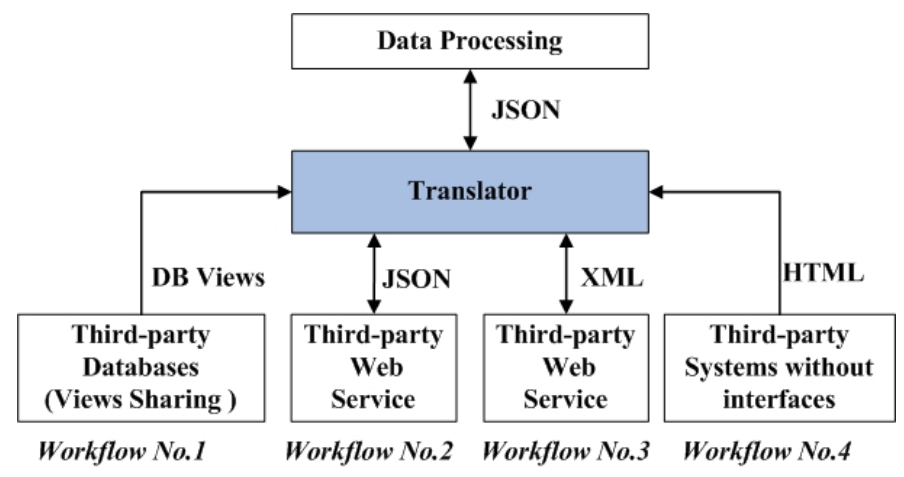

Figure 4. The Data integration and translation workflow. 


\section{Conclusion}

This paper describes why and how we design mobile campus portal to meet the requirements of educational big data environment. We introduced the topology, application and system structures of our MCP, and also discussed our implementation of 5Vs of big data concept in MCP.

After two-years running, the mobile campus portal has received positive feedbacks from users. It runs efficiently and stably, and provides a good information platform for constructing the mobile educational big data environment.

In the future, we will focus on the data analysis and data mining in mobile campus portal in order to extract more valuable information from big data.

\section{Acknowledgements}

This work is supported by the mobile campus construction project (a 985 project) of Xi'an Jiaotong University, China.

\section{References}

[1] Shi, Q. (2010) Design and Implementation of Mobile Leaning System Based on Mobile Phone. Lecture Notes in Electrical Engineering, 72, 365-369. http://dx.doi.org/10.1007/978-3-642-14350-2 46

[2] Setiabudi, D.H., Tjahyana, L.J. and Winsen (2013) Mobile Learning Application Based on Hybrid Mobile Application Technology Running on Android Smart-Phone and Blackberry. International Conference on ICT for Smart Society (ICISS), Jakarta, June 2013, 1-5.

[3] Self, R.J. (2014) Governance Strategies for the Cloud, Big Data, and Other Technologies in Education. 2014 IEEE/ ACM 7th International Conference on Utility and Cloud Computing (UCC), London, December 2014, 630-635. http://dx.doi.org/10.1109/ucc.2014.101

[4] Yu, Z., Liang, Y., Xu, B., et al. (2011) Towards a Smart Campus with Mobile Social Networking. IEEE Conference on Internet of Things and Cyber, Physical and Social Computing, Dalian, October 2011, 162-169.

[5] Aron, J.L. (2014) Sharing Best Practices for the Implementation of Big Data Applications in Government and Science Communities. IEEE International Conference on Big Data, Washington DC, October 2014, 8-10.

[6] Katal, A., Wazid, M. and Goudar, R.H. (2013) Big Data: Issues, Challenges, Tools and Good Practices. 2013 Sixth International Conference on Contemporary Computing (IC3), Noida, August 2013, 404-409. http://dx.doi.org/10.1109/ic3.2013.6612229

[7] Wehner, P., Piberger, C. and Go04Hringer D. (2014) Using JSON to Manage Communication between Services in the Internet of Things. International Symposium on Reconfigurable and Communication-Centric Systems-on-Chip (ReCoSoC), Montpellier, May 2014, 1-4.

[8] Michalik, P., Stofa, J. and Zolotova, I. (2014) Concept Definition for Big Data Architecture in the Education System. IEEE 12th International Symposium on Applied Machine Intelligence and Informatics (SAMI), Herl'any, January 2014, 331-334. http://dx.doi.org/10.1109/sami.2014.6822433

[9] http://www.sas.com/big-data/

[10] http://www-01.ibm.com/software/data/bigdata/

[11] http://drops.dagstuhl.de/opus/volltexte/2014/4562/

[12] Hubbard, J., Weimer, K. and Chen, Y. (2014) A Study of SSL Proxy Attacks on Android and iOS Mobile Applications. IEEE Conference on Consumer Communications and Networking, Las Vegas, January 2014, 86-91.

[13] You, W., Xu, L. and Rao, J. (2013) A Comparison of TCP and SSL for Mobile Security. International Conference on Sensor Network Security Technology and Privacy Communication System (SNS \& PCS), Harbin, May 2013, 206-209.

[14] Abura'ed, N., Otrok, H., Mizouni, R. and Bentahar, J. (2014) Mobile Phishing Attack for Android Platform. 2014 10th International Conference on Innovations in Information Technology, Al Ain, November 2014, 18-23. http://dx.doi.org/10.1109/innovations.2014.6987555 